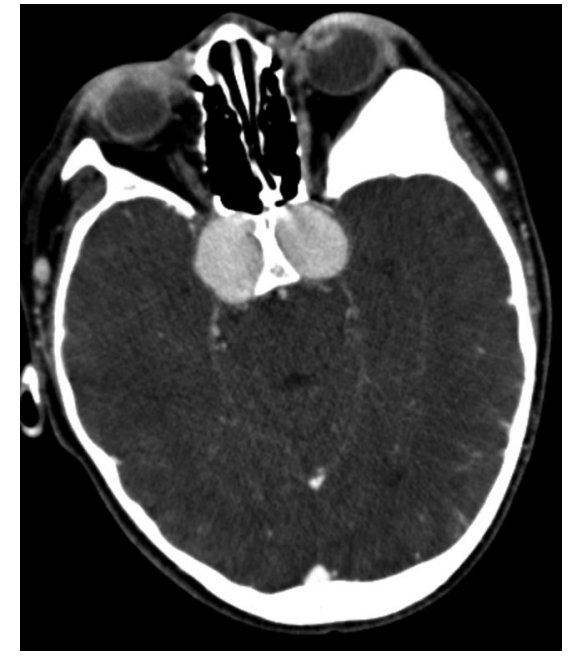

Fig 1. CT postcontrast, part of CTA, demonstrates bilateral marked enlargement of the bilateral cavernous internal carotid arteries.

\section{Bilateral Cavernous Internal Carotid Aneurysms in a Child with Juvenile Paget Disease and Osteoprotegerin Deficiency}

Juvenile Paget disease or familial hyperphosphatasia is a rare autosomal recessive disorder, which represents a distinct clinical condition, not simply a juvenile-onset form of Paget disease. Osteoprotegerin deficiency leads to marked osteoclast activation and greatly accelerated bone remodeling. We report a case of a child with a severe form of this condition and bilateral cavernous internal carotid artery aneurysms. The association suggests a role of osteoprotegerin deficiency in vessel wall abnormalities.

An 11-year-old boy presented with the chief complaint of difficulty looking straight with both eyes. Physical findings included strabismus of the left eye, developmentally short stature, bowed limbs, head disproportionately large for the trunk, and normal mental aptitude. Conventional noncontrast CT and CT angiography (CTA) of the head were performed. Symmetric aneurysms were demonstrated in the internal carotid arteries in the cavernous sinuses (Fig 1). There was remodeling and smooth thinning of the sphenoid bone between the aneurysms and the sphenoid sinus, abnormal definition of the inner ear structures bilaterally, and ground-glass changes within the medullary space of the calvaria. Conventional angiography confirmed the aneurysms (Fig 2).

Despite the name, juvenile Paget disease is distinct from the relatively common adult-onset disease known as Paget disease. Juvenile Paget disease, hereditary hyperphosphatasia or hyperostosis corticalis deformans juvenilis, is a rare autosomal recessive disease of bone, which presents in infancy or early childhood (OMIM \#239000; NCBI; www.ncbi.nlm.nih.gov/entrez/dispomim.cgi?id=239000). Accelerated remodeling of the skeleton results in bone deformities, pain secondary to fractures, osteopenia of the long bones, corticomedullary indistinctness, and coarsening of the trabecular bone. The juvenile disease affects the entire skeleton, whereas the conventional Paget disease involves more localized patterns of osteopathy, with both destructive and sclerotic phases. ${ }^{1}$ Juvenile Paget disease has been shown, in at least some cases, to be due to genetic deficiency of osteoprotegerin (not a known mechanism of conventional Paget disease). In some cases, including this patient, there is a homozygous deletion of a gene (TNFRSF11B) on chromosome 8q; the missing gene normally encodes osteoprotegerin. Past analysis had shown the patient presented here to have no detectable serum levels of osteoprotegerin. ${ }^{1}$

Osteoprotegerin, a soluble glycoprotein, acts as a decoy receptor that blocks the activation by receptor activator for nuclear factor $\kappa \mathrm{B}$ ligand (RANKL) of osteoclasts. The osteoprotegerin/RANKL/receptor activator of nuclear factor $\kappa \mathrm{B}$ system is central to regulation of osteoclast activity and bone turnover, and many cytokines and hormones affect this system. In addition to their role in a wide variety of skeletal disorders, osteoprotegerin and RANKL are being intensively studied for roles in the immune and vascular systems. ${ }^{2}$ For example, osteoprotegerin/RANKL balance may be a factor in atherosclerosis. Of particular interest in the current patient, osteoprotegerin has been shown in cell culture studies to be a factor promoting cell survival, protecting endothelial cells from apoptosis. ${ }^{3}$

Bilateral cavernous carotid artery aneurysms are clinically uncommon conditions. ${ }^{4}$ To our knowledge, the association of juvenile Paget disease with cerebral aneurysms has not previously been described. Regardless of the mechanism of vascular wall abnormality, the marked bone remodeling of juvenile Paget disease may additionally contribute to, or permit, the prominent size of the aneuryms and deformity of the skull base in the present patient. Without histologic evidence, it is not possible to characterize the precise nature of vascular abnormality in the patient presented here. However, the unusual
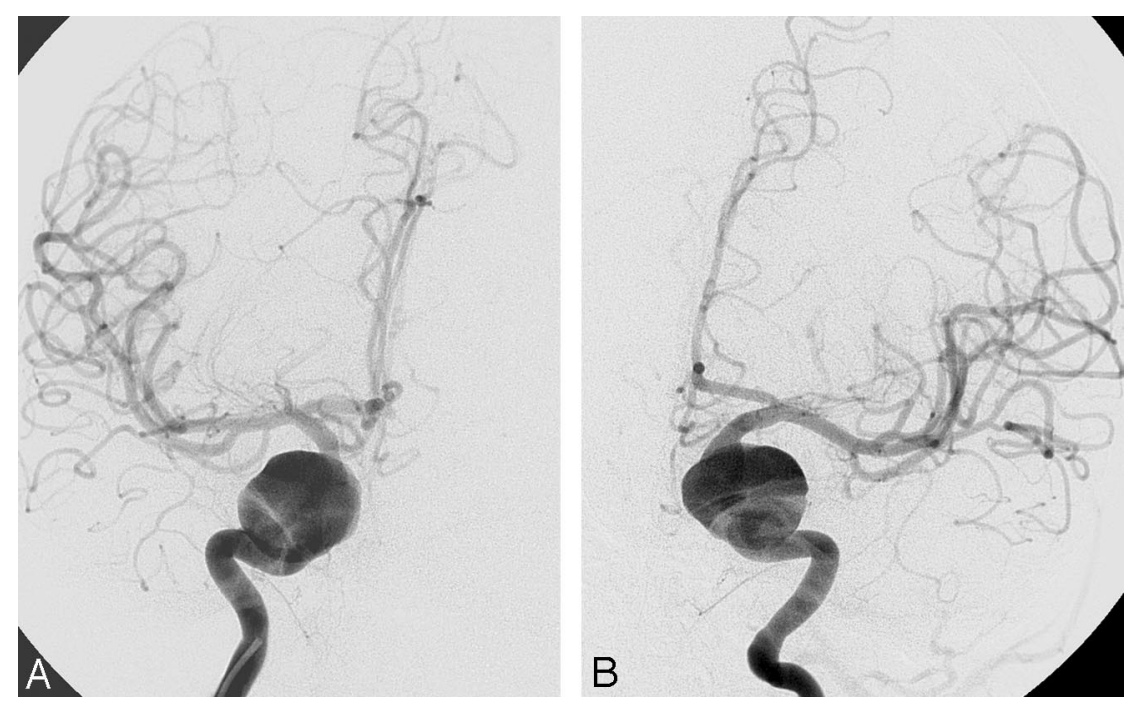

Fig 2. Conventional angiogram demonstrates giant bilateral cavernous internal carotid artery aneurysms, $A$, right, and $B$, left internal carotid artery injection. 
findings in a child with particularly severe juvenile Paget disease resulting from osteoprotegerin deficiency provide evidence for potential vascular complications in these patients in addition to severe osteopathy and raise further questions about the possible role of osteoprotegerin in preserving arterial wall integrity.

$$
\begin{array}{r}
\text { C.A. Allen } \\
\text { B.L. Hart } \\
\text { Department of Radiology } \\
\text { C.L. Taylor } \\
\text { Departments of Radiology and Neurosurgery } \\
\text { C.L. Clericuzio } \\
\text { Department of Pediatrics } \\
\text { University of New Mexico School of Medicine } \\
\text { Albuquerque, NM }
\end{array}
$$

\section{References}

1. Whyte MP, Obrecht SE, Finnegan PM, et al. Osteoprotegerin deficiency and juvenile Paget's disease. N Engl J Med 2002;347:175-84

2. Hofbauer LC, Schoppet M. Clinical implications of the osteoprotegerin/ RANKL/RANK system for bone and vascular diseases. JAMA 2004;292:490-95

3. Malyankar UM, Scatena M, Suchland KL, et al. Osteoprotegerin is an alpha vbeta 3-induced, NF-kappa B-dependent survival factor for endothelial cells. J Biol Chem 2000;275:20959-62

4. Linskey ME, Sekhar LN, Hirsch W, et al. Aneurysms of the intracavernous carotid artery: clinical presentation, radiographic features, and pathogenesis. Neurosurgery 1990;26:71-79

DOI 10.3174/ajnr.A0755 